\title{
Comparison of postural control between subgroups of persons with nonspecific chronic low back and healthy controls during the modified Star Excursion Balance Test
}

\author{
Amjad Shallan ${ }^{a, b}$, Everett Lohman ${ }^{a}$, Faris Alshammari ${ }^{b}$, Robert Dudley ${ }^{c}$, Omar Gharisia ${ }^{a}$, \\ Rana Al-Marzouki ${ }^{a}$, Helen Hsu ${ }^{a}$, Noha Daher ${ }^{d}$ \\ ${ }^{a}$ Department of Physical Therapy, School of Allied Health Professions, Loma Linda University, Loma Linda, CA, USA \\ bepartment of Physical Therapy, Faculty of Applied Medical Sciences, The Hashemite University, Zarqa, Jordan \\ ${ }^{\circ}$ Department of Kinesiology, School of Behavioral and Applied Sciences, Azusa Pacific University, Azusa, CA, USA \\ ${ }^{d}$ Department of Allied Health Studies, School of Allied Health Professions, Loma Linda University, Loma Linda, CA, USA
}

\begin{abstract}
Objective: To compare the postural control between non-specific chronic low back pain (NSCLBP) subgroups and healthy people during dynamic balance performance using a modified Star Excursion Balance Test (mSEBT).

Design: Cross-sectional study.

Methods: Eighteen NSCLBP subjects ( 9 active extension pattern [AEP], 9 flexion pattern [FP]), and 10 healthy controls were enrolled in this study. All subjects performed mSEBT on their dominant leg on a force plate. Normalized reach distance and balance parameters, including the center of pressure (COP) displacement and velocity, were recorded.

Results: There were significant differences in mean reach distances in both posterolateral and posteromedial (PM) reach directions between AEP and healthy subjects $(p<0.001)$ and between FP and healthy subjects $(p<0.001)$. However, there were no significant differences among the three groups in the anterior reach direction. Also, the results showed no significant differences in mean COP variables (velocity and displacement) between pooled NSCLBP and healthy subjects. However, the subjects were reclassified into AEP, FP and healthy groups and the results showed a significant difference in mean COP velocity in the PM direction between AEP and FP subjects ( $p=0.048)$, and between AEP and healthy subjects $(p=0.024)$.

Conclusions: The findings in this study highlight the heterogeneity of the individuals with NSCLBP and the importance of identifying the homogenous subgroups. Individuals with AEP and FP experience deficits in dynamic postural control compared to healthy controls. In addition, the findings of this study support the concept of the Multidimensional Classification System.
\end{abstract}

Key Words: Human body, Low back pain, Musculoskeletal diseases, Postural balance, Posture

\section{Introduction}

Low back pain (LBP) is one of the most common musculoskeletal disorders with more than $80 \%$ of individuals experiencing LBP at one time in their life [1]. Nonspecific chronic low back pain (NSCLBP) is considered to be one of the most common LBP classifications [2]. NSCLBP is defined as LBP for more than three months without known specific sources of pain and with no evidence of pathoanatomic and abnormality with imaging [3]. However, NSCLBP could result from different factors, such as biomechanical, psychosocial, and genetic factors or the interactions between some or all of them [4]. In addition, NSCLBP is considered as a disabling condition that limits daily activities of the affected people [5]. Therefore, understanding the mechanism of NSCLBP disorders may assist healthcare providers

Received: 20 June, 2019 Revised: 7 July, 2019 Accepted: 9 July, 2019

Corresponding author: Amjad Shallan (ORCID https://orcid.org/0000-0002-2669-8780)

Department of Physical Therapy, Loma Linda University, 24851 Circle Dr, Loma Linda, CA 92354, USA

Tel: 1-909-558-1000, ext. 83171 Fax: 1-909-558-0459 E-mail: ashallan@llu.edu

(c) This is an Open-Access article distributed under the terms of the Creative Commons Attribution Non-Commercial License (http://creativecommons.org/licenses/ by-nc/4.0) which permits unrestricted non-commercial use, distribution, and reproduction in any medium, provided the original work is properly cited.

Copyright (๑ 2019 Korean Academy of Physical Therapy Rehabilitation Science 
to develop proper interventions.

Postural control is required to safely and effectively perform a wide range of daily activities [6]. Postural control is defined as the ability of the human body to maintain the center of gravity within the base of support [7]. However, studies have identified postural control changes in people with LBP especially in terms of center of pressure (COP) parameters (e.g., COP velocity and displacement) $[8,9]$.

Numerous factors may contribute to postural control alterations in people with NSCLBP [10]. Deficits in the neuromusculoskeletal systems, such as a reduction in somatosensory input, processing, or motor output, have been found to contribute to alterations in postural control in people with NSCLBP [11]. In addition, studies revealed that LBP could affect postural stability through numerous co-existing factors such as pain, alteration in movement strategies, and fear of pain [10].

Different methods are used to detect postural control and dynamic balance deficits. However, many of these methods are complicated and costly [12]. The Star Excursion Balance Test (SEBT) is a simple tool that has been used to measure functional and dynamic balance [13]. The SEBT has been used to detect dynamic balance impairments that may lead to lower extremity injuries $[14,15]$. Recently, several studies have utilized the SEBT to detect dynamic balance impairments in people with LBP $[13,16]$. Also, SEBT is considered a challenging task for people with LBP. Therefore, the SEBT may provide clinicians with valuable information regarding postural control impairments and movement strategies in people with LBP [17]. The modified version of the SEBT ( $\mathrm{mSEBT}$ ) is used to reduce the potential fatigue effect and the redundancy among the eight directions in the original SEBT [18]. The mSEBT consists of three directions including the anterior, posteromedial and posterolateral directions. The mSEBT has shown to have excellent interrater reliability and strong intra-rater and test-retest reliability in detecting dynamic balance impairments $[19,20]$.

The force plate has been used to quantify the COP oscillations during static and dynamic postural control in people with LBP $[9,10]$. Despite the large number of studies investigating postural stability in people with LBP, the results have been inconsistent with contradictory findings [9,10]. One reason behind these inconsistencies may be related to the complexity and heterogeneity of people with LBP [9,21]. Therefore, classifying people with NSCLBP into subgroups, according to the type of dysfunction, may be important in order to identify the adaptive postural control strategies within each subgroup [22].

Attempts have been made to classify individuals with NSCLBP [23,24]. One of these classification systems is the Multidimensional Classification System (MDCS) [24]. The MDCS outlines five motor control impairment (MCI) subgroups with the flexion pattern (FP) and active extension pattern (AEP) being the most common in the clinical setting [25,26]. Based on O'Sullivan [24], MCI subgroups exhibit full range of motion (ROM) in the direction of pain provocation. Also, MCI subgroups utilize modifications in body postures and movement strategies to deal with the expected pain.

Previous studies have investigated the physical characteristics between these two MCI subgroups (AEP and FP) and healthy subjects in terms of kinematics and muscle activity during static and functional tasks [27-29]. However, there is limited information about postural control and dynamic balance characteristics in these subgroups. Therefore, the purpose of this study was to examine postural control and dynamic balance performance between MCI subgroups (FP and AEP) compared to the healthy subjects using the mSEBT. The mSEBT is considered an extension activity that is based on the MDCS and include aggravating factors in AEP. Therefore, we hypothesized that postural control and dynamic balance during performance of $\mathrm{mSEBT}$ would be different in AEP subjects.

\section{Methods}

\section{Subjects}

A total of 28 subjects participated in this study from Loma Linda University Medical Health and the surrounding community. Subjects were recruited using fliers. LBP subjects were included in the study if they were between 18 and 60 years old, had LBP for more than 3 months, and the pain was localized to the low back and/or buttock regions only. The control subjects were healthy individuals who have been free of LBP for at least two years and have similar characteristics to subjects with LBP. The exclusion criteria for both groups were: signs of serious spinal pathology, fracture, malignancy, history of spinal surgery, lower extremity injury in the previous two years, vestibular dysfunction, or balance disorders. In addition, females were excluded from the study if they were breastfeeding or pregnant (self-reported) to avoid potential complications or side effects. 


\section{Measurement procedure}

All tests were performed at the Physical Therapy Department in the School of Allied Health Professions, Loma Linda University, CA, USA. Data collection took approximately 60 minutes to complete. The study protocol and procedures were explained to the subjects in details by the primary researcher. After that, all subjects read and signed the informed consent. Then, demographic data such as age, weight, height and dominant leg, defined at the limb used to kick a ball, were obtained prior to the data collection session. All subjects completed a medical history questionnaire and the International Physical Activity Questionnaire-Short Form to measure the physical activity level. Subjects in the LBP groups were asked to report the measures for pain using the Visual Analogue Scale, disability levels caused by LBP using the Roland-Morris Disability Questionnaire and the presence of pain-related fear of movement using the Tampa Scale of Kinesiophobia (Table 1).

\section{$\mathrm{MCl}$ subgroup classification}

AEP and FP were chosen in this study because of their high prevalence $[25,26]$. To establish MCI subgroups classification (AEP and FP), comprehensive subjective and objective assessments were conducted. In the subjective assessment, the full history of the subject's LBP was taken as well as the pain behaviors, such as the easing and aggravating postures and activities. In the objective examination, the battery of postures and spinal ROM were observed. In addition, usual standing and sitting, full trunk flexion, extension, and side bending were evaluated. Finally, the Passive Physiological Intervertebral Movements at, above, and below the provoking lumbar segment were performed to assess the existence of joint hypo-mobility or hypermobility [28]. Previous research has identified that clinicians have good inter-rater reliability in applying the subclassification system $[30,31]$. Therefore, MCI subgroups (AEP and FP) subjects were examined and classified independently by two physical therapists based on MDCS criteria [24], and only subjects who had an agreement of both clinicians were included in the study.

\section{Test description}

The mSEBT is a measure of dynamic balance [18]. The original version consists of eight strips of tape placed at $45^{\circ}$ angles to each other from the center of a grid. The subject stands on one leg at the center of the "star" created by the intersection of the tape pieces. Each strip of tape is labeled based on the excursion direction relative to the stance leg. Hertel et al. [18] found that there is considerable redundancy among the eight directions of the original SEBT and they proposed a modified version of the SEBT which was used in this study. The mSEBT consists of three directions including the anterior, posteromedial, and posterolateral directions. Strong intra-rater and test-retest reliability have been reported regarding this tool $[19,32]$. In addition, the predictive and construct validity of the test was supported in previous studies [18,33].

\section{Data collection procedures}

Verbal and visual demonstration of proper performance of the mSEBT were provided to the subjects. Then, the subjects were instructed to align the lateral malleolus of the dominant leg at the intersection point of the three directions with the

Table 1. Mean (SD) of baseline characteristics by study subgroups

$(\mathrm{N}=28)$

\begin{tabular}{|c|c|c|c|c|}
\hline \multirow{2}{*}{ Characteristics } & \multicolumn{4}{|c|}{ Study subgroups } \\
\hline & $\operatorname{AEP}(n=9)$ & $\mathrm{FP}(\mathrm{n}=9)$ & Healthy $(n=10)$ & $p$-value \\
\hline Female $^{b}$ & $5(55.6)$ & $8(88.9)$ & $7(70.0)$ & 0.290 \\
\hline Age (y) & $28.8(5.0)$ & $27.2(3.6)$ & $26.8(2.6)$ & 0.510 \\
\hline BMI $\left(\mathrm{kg} / \mathrm{m}^{2}\right)$ & $25.8(5.0)$ & $23.5(2.7)$ & $23.8(3.0)$ & 0.370 \\
\hline Physically active $^{\mathrm{b}}$ & $9(100.0)$ & $9(100.0)$ & $9(90.0)$ & 0.760 \\
\hline Pain level ${ }^{\mathrm{c}}$ & $2.8(1.6)$ & $4.4(2.0)$ & - & 0.070 \\
\hline $\mathrm{TSK}^{\mathrm{c}}$ & $34.9(8.2)$ & $37.3(3.7)$ & - & 0.430 \\
\hline $\mathrm{RMQ}^{\mathrm{c}}$ & $5.6(0.9)$ & $5.4(0.5)$ & - & 0.750 \\
\hline
\end{tabular}

Values are presented as $\mathrm{n}(\%)$ or mean $(\mathrm{SD})$.

AEP: active extension pattern, FP: flexion pattern, BMI: body mass index, TSK: tampa scale for kinesiophobia, RMQ: Roland-Morris Disability Questionnaire, - : not available.

${ }^{a}$ One-way analysis of variance. ${ }^{b}$ Chi Square test of independence. ${ }^{c}$ Independent t-test. 
foot oriented toward the anterior direction with their hands placed on their hips. After that, the subjects were instructed to reach as far as possible with the non-stance leg and pointing with their big toe to the marked tape and return to the starting position [34]. Subjects performed 6 practice trials prior to the actual test trials to minimize the learning effect [19]. Next, the three test trials were recorded in each direction (Anterior, PM and PL) with 15-second rest periods between each trial [18]. The subjects performed the mSEBT on the force plate without wearing shoes to eliminate the influence of varying footwear (Figure 1) [35]. The trial was considered invalid if one of the following situations occurred; the subjects removed their hands off of their hips, the heel of stance limb lost contact with the ground during reaching, the subject put weight onto their reaching foot on the ground, or lost their balance during reach out or return [36]. The leg length (from the anterior superior iliac spine to the medial malleolus) was measured with the subject in supine lying [36]. This measurement was used in normalizing the mSEBT reach distance for each subject [37]. The maximum reaching distance in every direction was normalized as a percentage of the stance limb length using this equation; maximum reach divided by leg length and the results were multiplied by 100 . The mean value of normalized reach in each direction was calculated for analysis [36].

\section{Data analysis}

A single force plate (AMTI Optim; Advanced Mechanical Technology Inc., Watertown, MA, USA) was used to evaluate the postural control parameters. The COP data were sampled at $2,000 \mathrm{~Hz}$ and force plate movements were described as the following: Antero-posterior movement was represented by the Y-axis, while the medio-lateral (ML) movement was represented by the $\mathrm{X}$-axis. ML and anteriorposterior (AP) displacements and velocity of COP were used for analysis. Visual 3D software (C-Motion Inc., Rockville, MD, USA) was used for raw data processing and analysis. COP data was filtered using a fourth order low-pass Butterworth filter with a cut off frequency of $5 \mathrm{~Hz}$.

\section{Statistical analysis}

Large effect sizes were reported in prior SEBT studies in participants with knee and ankle disorders [15,35]. The sample size was determined using $\alpha=0.05$, power $=0.80$, and an effect size $\mathrm{f}=0.65$. According to power analysis, nine subjects are required in each group.

Data were summarized using mean and standard deviation for quantitative variables and counts (\%) for qualitative variables. The normality of continuous variables was examined using Shapiro Wilk's test and Box plots. The characteristics of the subjects were compared among the study groups using chi-square for qualitative variables, and one-way ANOVA or independent t-test for quantitative variables.

Mean outcome variables were compared among the three groups (FP, AEP, and healthy) using one-way ANOVA. If the results of the test were statistically significant, post hoc testing using Bonferroni test was conducted. The level of significance was set at alpha $=0.05$. Statistical analysis was

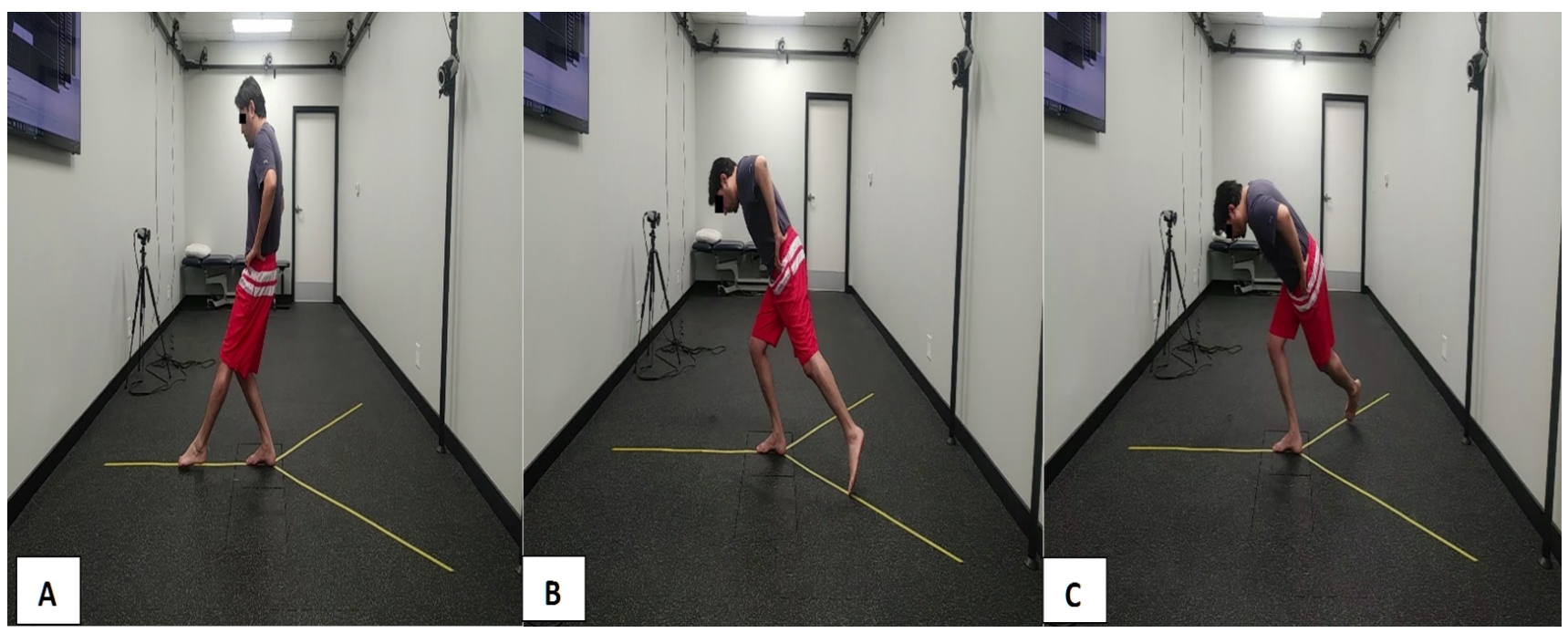

Figure 1. The modified Star Excursion balance Test. Subject reaches in the (A) anterior, (B) posteromedial, and (C) posterolateral directions. 
performed using IBM SPSS Software version 25 for Windows (Chicago, IL, USA).

\section{Results}

A total of 28 subjects (18 with LBP and 10 healthy) with a mean age of $27.6 \pm 3.8$ years and body mass index $24.3 \pm 3.7$ $\mathrm{kg} / \mathrm{m}^{2}$ participated in the study. The demographic characteristics of the subjects by the study group are shown in Table 1 . There were no significant differences in the characteristics among the three groups ( $p>0.05)$. In addition, results showed that there was no significant difference in baseline characteristics between pooled NSCLBP and healthy subjects ( $p>0.05$, Table 1).

There was no significant difference in mean reach distance among the three groups $\left(\mathrm{F}_{2,27}=1.0, p=0.38, \eta^{2}=0.07\right)$ in the anterior direction. However, there was a significant difference in the mean reach distance in the PL and PM directions, by study group $\left(\mathrm{F}_{2,27}=17.6, p<0.001, \eta^{2}=0.58\right.$, and $\mathrm{F}_{2,27}=9.3, p<0.001, \eta^{2}=0.43$, respectively). In the PL direction, there was a significant difference in mean reach distance between AEP and healthy (73.4 \pm 8.4 vs. $90.7 \pm 5.2, p<$ $0.001)$, and FP and healthy (75.4 \pm 7.3 vs. $90.7 \pm 5.2, p<0.001)$. Similarly, in the PM direction, there was a significant difference in mean reach distances between AEP and healthy (81.3 \pm 10.9 vs. $93.3 \pm 4.5, p=0.018)$, and FP and healthy ( $76.7 \pm 9.8$ vs. $93.3 \pm 4.5, p=0.001)$. However, there was no significant difference in mean reach distances between AEP and FP in PL and PM directions ( $p>0.05$, Figure 2).

The results showed no significant differences in mean COP variables (velocity and displacement) between pooled NSCLBP and healthy subjects (Table 2). However, when the subjects were classified into AEP, FP and healthy groups, the results showed a significant difference in mean AP COP velocity in the PM direction between AEP and FP subjects (71.2 \pm 17.2 vs. $56.4 \pm 9.3, p=0.048)$, and between AEP and healthy subjects $(71.2 \pm 17.2$ vs. $55.1 \pm 8.5, p=0.024)$ (Table 3$)$.

\section{Discussion}

This study examined the differences in the mSEBT scores in two subgroups of NSCLBP compared with healthy subjects. In addition, it examined the dynamic postural control using the COP parameters during the performance of the mSEBT. We hypothesized that postural control and dynamic balance during performance of mSEBT would be different in AEP subjects. The results supported our hypothesis and there were significant differences in mean reach distances in both posterolateral and posteromedial reach directions between AEP and healthy, and between FP and healthy subjects. Also, there was a significant difference in mean COP velocities in the posteromedial direction between AEP and FP subjects, and between AEP and healthy subjects. In addition, our findings validate the MCI subclassification and provide more evidence regarding postural control compensatory strategies that may occur in these subgroups of individuals with NSCLBP. To our knowledge, this is the first study to examine the dynamic postural control deficits in people with NSCLBP by subgroups using the modified SEBT.

The results of this study indicated that the reach distances in the PL and PM directions were significantly lower in both AEP and FP groups compared to healthy group. However, there was no significant difference in mean reach distance in the anterior direction among the three groups. Subjects in both AEP and FP subgroups may have a limited pelvic anterior tilt compared to healthy subjects, which leads to de-

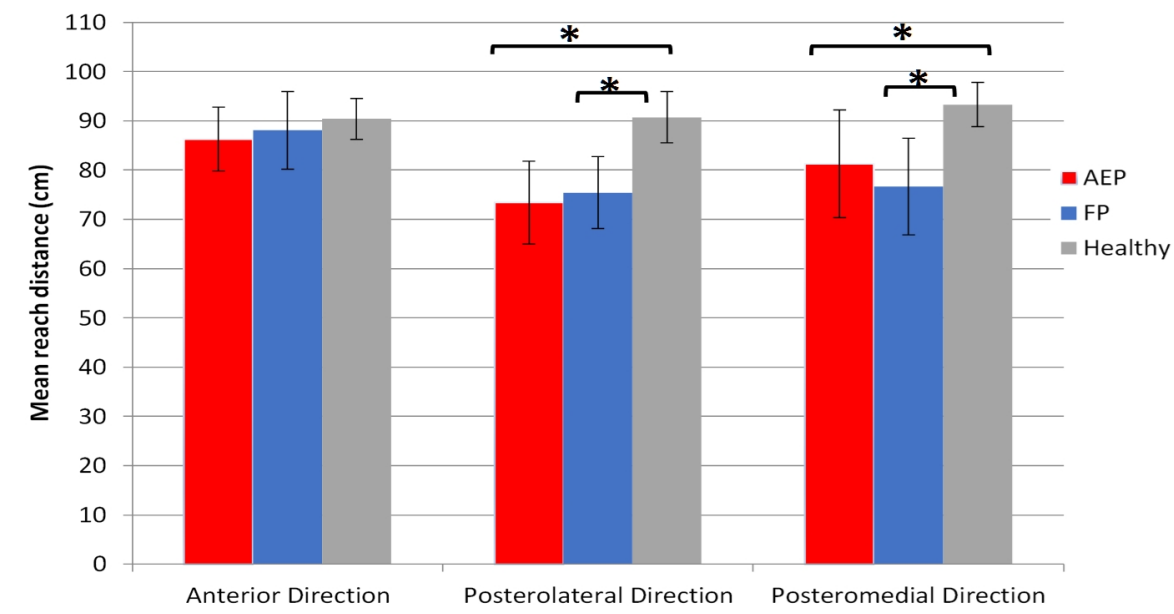

Figure 2. Mean reach distance $(\mathrm{cm})$ by study group ( $\mathrm{N}=28)$. AEP: active extension pattern, FP: flexion pattern. *Significant difference $(p<0.05)$. Values are presented as mean (SD). 
Table 2. Comparison of COP displacement and velocity between pooled LBP and healthy subjects

$(\mathrm{N}=28)$

\begin{tabular}{|c|c|c|c|c|c|}
\hline \multirow{2}{*}{ Direction } & \multirow{2}{*}{ COP parameter } & Pooled LBP $(n=18)$ & Healthy $(n=10)$ & \multirow{2}{*}{ Cohen's d } & \multirow{2}{*}{$p$-value } \\
\hline & & Mean (SD) & Mean (SD) & & \\
\hline \multirow[t]{6}{*}{ Anterior } & Velocity $(\mathrm{mm} / \mathrm{s})$ & & & & \\
\hline & AP & $64.2(15.2)$ & $64.8(16.7)$ & 0.040 & 0.930 \\
\hline & ML & $40.6(11.8)$ & $37.5(6.8)$ & 0.300 & 0.459 \\
\hline & Displacement (mm) & & & & \\
\hline & AP & $245.4(54.2)$ & $275.4(59.8)$ & 0.540 & 0.187 \\
\hline & ML & $170.8(48.7)$ & $192.0(72.6)$ & 0.370 & 0.364 \\
\hline \multirow[t]{6}{*}{ Posterolateral } & Velocity $(\mathrm{mm} / \mathrm{s})$ & & & & \\
\hline & AP & $60.1(14.0)$ & $62.1(11.6)$ & 0.150 & 0.713 \\
\hline & ML & $50.0(12.9)$ & $51.3(9.5)$ & 0.110 & 0.786 \\
\hline & Displacement (mm) & & & & \\
\hline & AP & $234.3(65.6)$ & $263.3(19.6)$ & 0.540 & 0.096 \\
\hline & ML & $216.6(55.5)$ & $249.2(79.7)$ & 0.510 & 0.214 \\
\hline \multirow[t]{6}{*}{ Posteromedial } & Velocity $(\mathrm{mm} / \mathrm{s})$ & & & & \\
\hline & $\mathrm{AP}$ & $63.8(15.4)$ & $55.1(8.5)$ & 0.660 & 0.065 \\
\hline & ML & $45.6(15.4)$ & $46.4(10.9)$ & 0.060 & 0.886 \\
\hline & Displacement (mm) & & & & \\
\hline & AP & $243.6(68.5)$ & $263.7(80.5)$ & 0.280 & 0.491 \\
\hline & ML & $198.6(66.8)$ & $231.1(67.2)$ & 0.490 & 0.230 \\
\hline
\end{tabular}

COP: center of pressure, LBP: low back pain, AP: anterior-posterior; ML: medio-lateral.

${ }^{a}$ Independent t-test.

Table 3. Comparison of COP displacement and velocity among the three groups

$(\mathrm{N}=28)$

\begin{tabular}{|c|c|c|c|c|c|c|}
\hline \multirow{2}{*}{ Direction } & \multirow{2}{*}{$\mathrm{COP}$ parameter } & $\operatorname{AEP}(n=9)$ & $\mathrm{FP}(n=9)$ & Healthy $(n=10)$ & \multirow{2}{*}{$\eta^{2}$} & \multirow{2}{*}{$p$-value ${ }^{\mathrm{a}}$} \\
\hline & & Mean (SD) & Mean (SD) & Mean (SD) & & \\
\hline \multirow[t]{6}{*}{ Anterior } & Velocity $(\mathrm{mm} / \mathrm{s})$ & & & & & \\
\hline & $\mathrm{AP}$ & $67.2(18.4)$ & $61.2(11.4)$ & $64.8(16.7)$ & 0.026 & 0.721 \\
\hline & ML & $40.7(12.0)$ & $40.5(12.3)$ & $37.5(6.8)$ & 0.021 & 0.764 \\
\hline & Displacement (mm) & & & & & \\
\hline & AP & $244.4(53.2)$ & $246.4(58.3)$ & $275.4(59.8)$ & 0.066 & 0.425 \\
\hline & ML & $160.8(66.2)$ & $180.8(20.9)$ & $192.0(72.6)$ & 0.052 & 0.515 \\
\hline \multirow[t]{6}{*}{ Posterolateral } & Velocity $(\mathrm{mm} / \mathrm{s})$ & & & & & \\
\hline & AP & $62.3(17.6)$ & $58.0(9.8)$ & $62.1(11.6)$ & 0.024 & 0.737 \\
\hline & ML & $52.6(12.0)$ & $47.5(13.9)$ & $51.3(9.5)$ & 0.035 & 0.643 \\
\hline & Displacement (mm) & & & & & \\
\hline & AP & $233.2(61.6)$ & $235.4(73.2)$ & $263.3(19.6)$ & 0.066 & 0.425 \\
\hline & ML & $221.8(53.8)$ & $211.4(60.1)$ & $249.2(79.7)$ & 0.063 & 0.444 \\
\hline \multirow[t]{6}{*}{ Posteromedial } & Velocity $(\mathrm{mm} / \mathrm{s})$ & & & & & \\
\hline & $\mathrm{AP}$ & $71.2(17.2)$ & $56.4(9.3)$ & $55.1(8.5)$ & 0.285 & $0.015^{\mathrm{b}}$ \\
\hline & ML & $49.4(15.1)$ & $41.8(15.6)$ & $46.4(10.9)$ & 0.051 & 0.519 \\
\hline & Displacement (mm) & & & & & \\
\hline & AP & $245.9(57.6)$ & $241.3(81.5)$ & $263.7(80.5)$ & 0.019 & 0.786 \\
\hline & ML & $185.4(57.3)$ & $211.8(76.3)$ & $231.1(67.2)$ & 0.081 & 0.350 \\
\hline
\end{tabular}

COP: center of pressure, AEP: active extension pattern, FP: flexion pattern, $\eta$ : effect size, AP: anterior-posterior, ML: medio-lateral. ${ }^{\mathrm{a}}$ One-way analysis of variance. ${ }^{\mathrm{b}}$ Significant difference between AEP and FP and between AEP and Healthy $(p<0.05)$.

crease in the PL and PM reaching distance [38]. Also, reaching in posterior directions in the $\mathrm{mSEBT}$ are more challenging compared to anterior reaching due to excessive lumbar lordosis that is required to finish the task which stresses the postural control system in NSCLBP groups to a point that limits the subjects' reach [39]. In addition, people with 
NSCLBP are more dependent on visual feedback due to altered proprioceptive input [40]. Reaching in posterior direction requires subjects to rely on proprioceptive input and the vestibular system to maintain the single leg balance compared to reaching forward where the subjects can use their vision for assistance. Therefore, there was no significant difference in reaching forward among groups [41].

Another explanation could be related to the pain avoidance behavior in both AEP and FP subjects [24,42]. Subjects in both MCI groups may anticipate pain during posterior reach which may lead them to avoid performing the task vigorously and consequently this results in poor performance in mSEBT in PM and PL directions compared to healthy subjects [39].

These findings are consistent with the Hooper et al. [43] study, that found significant differences in reach distances between the LBP subgroups (current LBP vs. LBP history) compared to healthy subjects in the PL and PM directions but not in the anterior direction. On the other hand, Ganesh et al. [13] found that people with LBP have a significant decrease in reach distances in the PM, PL, and the anterior directions. However, Appiah-Dwomoh et al. [36] did not find any significant differences in any reach directions between healthy athletes and athlete with LBP. The inconsistency in the findings of the above studies can be explained by many factors. First, the heterogeneity of the LBP subjects in the previous studies may lead to the differences in the postural stability strategies that each subject used to maintain their balance. In other words, findings in one subgroup of subjects were counteracted by other subgroups when the people with NSCLBP were studied heterogeneously (the washout effect phenomenon) [44]. Second, LBP subjects' characteristics, such as age and physical activities were different which may contribute to these differences in the results [45],

Our results showed no significant difference between the pooled NSCLBP and healthy subjects in mean COP measures (displacement and velocity). After subgrouping NSCLBP subjects into FP and AEP groups, the results showed a higher mean COP sway velocity in AEP subjects compared to the FP and healthy subjects in PM direction. This finding confirms the presence of washout effect and establishes the need for studying the homogeneous subgroups of NSCLBP in order to better understand the NSCLBP disorder [46].

Our findings support the findings by Seraj et al. [21], who found no significant differences in postural control variables between the pooled NSCLBP subjects and healthy subjects during lifting task. However, when NSCLBP subjects were classified into AEP and FP, the results revealed that AEP subjects had a significant difference in postural control compared to FP and healthy subjects during lifting task.

In our study, AEP subjects had a higher sagittal COP velocity as compared to FP and healthy subjects during PM direction of $\mathrm{mSEBT}$. One of the reasons behind this finding may be the nature of the required task. Reaching in the PM direction requires anterior pelvic tilt and stresses lumbar spine resulting in excessive lordosis or hyperextension of lumbar spine. Based on the MCI classification, the standing and extension positions are more likely to aggravate pain in the AEP group as compared to the FP group [26]. According to the pain adaptation model, the normal response of the body is to increase paraspinal muscle activity in the AEP subjects, which may increase the load on the trunk structure [46]. These changes in proprioception and the muscle activity may result in more postural sway velocity in the AEP subjects as compared to the FP and the healthy subjects.

Subjects in the AEP group will tend to move slower in the PM direction as pain-avoidance behavior to finish the task with less pain. Slower movement in the PM direction will result in longer duration of the single leg stance and more activation of the lumbar extensor muscles resulting in fatigue which leads to the increase in body sway [47]. As noted earlier, the subjects in this study were young. Therefore, the nervous system will have a faster reaction in order to correct body sway, and to maintain stability. According to Newton's third law, each action has a reaction that is equal in magnitude and opposite in direction. Also, according to the pendulum theory, anterior acceleration will be corrected by posterior acceleration, which results in body sway. Since the correction of body sway was fast, we expect that the repeated sway action will be fast as well resulting in the increase in COP sway velocity.

We did not find any significant differences between the FP and the healthy subjects in COP displacement and velocity, suggesting that the FP and the healthy subjects may adopt similar strategies for postural control during the dynamic balance test [46]. Also, it could be that the mSEBT was not challenging enough to aggravate the pain in the FP group to exhibit different postural control strategies compared to the healthy subjects. In addition, it is expected to have no significant difference in the mean displacement of COP among the groups due to the fact that all subjects were young and physically active [36].

This study presents several unique contributions to the 
LBP literature. First, this study was the first to examine mSEBT in homogenous subgroups of NSCLBP (AEP and FP) and compare them to healthy subjects. In addition, our results showed that reaching distance alone is not enough to show the whole picture of the postural control deficits in NSCLBP subgroups, and it is important to investigate other variables, such as COP velocity in order to identify the postural control deficits those population.

There were some limitations in this study. First, the sample size was small requiring future research needs to recruit a larger sample size to investigate the postural stability differences between NSCLBP subgroups. Second, the pain and disability level in NSCLBP subgroups were relatively low. Subjects with NSCLBP with high levels of pain and disability may exhibit different postural stability strategies. Third, trunk muscle activation or trunk kinematics was not measured. This information could assist in better understanding of the compensatory movement patterns that each subgroup uses during dynamic balance. In addition, the number of the subjects in pooled NSCLBP was higher than healthy subjects. Therefore, this should be put into consideration during interpretations the findings of this study.

In conclusion, the findings in this study highlight the heterogeneity of the subjects with NSCLBP and the importance of identifying the homogenous subgroups. The findings showed that the dynamic balance and postural control were significantly different between AEP and FP, and AEP and healthy subjects during dynamic balance using the mSEBT. The AEP subjects exhibited more body sway velocity in the posteromedial direction of the mSEBT. However, there were no significant differences observed between FP and healthy subjects, suggesting that FP and healthy individuals may adopt similar postural control strategies during dynamic balance. Clinically, the mSEBT should be incorporated into a NSCLBP rehabilitation program to evaluate dynamic balance and monitor rehabilitation progression of NSCLBP homogenous subgroups.

\section{Acknowledgements}

The authors would like to thank Prarthana Dhotre for her assistance in data collection.

\section{Conflict of Interest}

The authors declared no potential conflicts of interest with respect to the authorship and/or publication of this article.

\section{References}

1. Walker BF, Muller R, Grant WD. Low back pain in Australian adults: prevalence and associated disability. J Manipulative Physiol Ther 2004;27:238-44.

2. Freburger JK, Holmes GM, Agans RP, Jackman AM, Darter JD, Wallace AS, et al. The rising prevalence of chronic low back pain. Arch Intern Med 2009;169:251-8.

3. Hart LG, Deyo RA, Cherkin DC. Physician office visits for low back pain. Frequency, clinical evaluation, and treatment patterns from a U.S. national survey. Spine (Phila Pa 1976) 1995;20:11-9.

4. Balagué F, Mannion AF, Pellisé F, Cedraschi C. Non-specific low back pain. Lancet 2012;379:482-91.

5. Vos T, Flaxman AD, Naghavi M, Lozano R, Michaud C, Ezzati $\mathrm{M}$, et al. Years lived with disability (YLDs) for 1160 sequelae of 289 diseases and injuries 1990-2010: a systematic analysis for the Global Burden of Disease Study 2010. Lancet 2012;380: 2163-96.

6. Maribo T, Schiøttz-Christensen B, Jensen LD, Andersen NT, Stengaard-Pedersen K. Postural balance in low back pain patients: criterion-related validity of centre of pressure assessed on a portable force platform. Eur Spine J 2012;21:425-31.

7. Winter DA. Biomechanics and motor control of human movement. Hoboken: John Wiley \& Sons; 2009.

8. Rainville J, Smeets RJ, Bendix T, Tveito TH, Poiraudeau S, Indahl AJ. Fear-avoidance beliefs and pain avoidance in low back pain--translating research into clinical practice. Spine J 2011;11:895-903

9. Mazaheri M, Coenen P, Parnianpour M, Kiers H, van Dieën JH. Low back pain and postural sway during quiet standing with and without sensory manipulation: a systematic review. Gait Posture 2013;37:12-22.

10. Ruhe A, Fejer R, Walker B. Center of pressure excursion as a measure of balance performance in patients with non-specific low back pain compared to healthy controls: a systematic review of the literature. Eur Spine J 2011;20:358-68.

11. Luoto S, Aalto H, Taimela S, Hurri H, Pyykkö I, Alaranta H. One-footed and externally disturbed two-footed postural control in patients with chronic low back pain and healthy control subjects. A controlled study with follow-up. Spine (Phila Pa 1976) 1998;23:2081-9; discussion 2089-90.

12. Ruhe A, Fejer R, Walker B. The test-retest reliability of centre of pressure measures in bipedal static task conditions--a systematic review of the literature. Gait Posture 2010;32:436-45.

13. Ganesh GS, Chhabra D, Mrityunjay K. Efficacy of the star excursion balance test in detecting reach deficits in subjects with chronic low back pain. Physiother Res Int 2015;20:9-15.

14. Linens SW, Ross SE, Arnold BL, Gayle R, Pidcoe P. Posturalstability tests that identify individuals with chronic ankle instability. J Athl Train 2014;49:15-23.

15. Herrington L, Hatcher J, Hatcher A, McNicholas M. A comparison of Star Excursion Balance Test reach distances between ACL deficient patients and asymptomatic controls. Knee 2009; 16:149-52.

16. Ganesh GS, Chhabra D, Pattnaik M, Mohanty P, Patel R, 
Mrityunjay K. Effect of trunk muscles training using a star excursion balance test grid on strength, endurance and disability in persons with chronic low back pain. J Back Musculoskelet Rehabil 2015;28:521-30.

17. Appiah-Dwomoh EK, Müller S, Mayer F. Reproducibility of static and dynamic postural control measurement in adolescent athletes with back pain. Rehabil Res Pract 2018;2018:8438350.

18. Hertel J, Braham RA, Hale SA, Olmsted-Kramer LC. Simplifying the star excursion balance test: analyses of subjects with and without chronic ankle instability. J Orthop Sports Phys Ther 2006;36:131-7.

19. Hertel J, Miller SJ, Denegar CR. Intratester and intertester reliability during the Star Excursion Balance Tests. J Sport Rehabil 2000;9:104-16.

20. Kinzey SJ, Armstrong CW. The reliability of the star-excursion test in assessing dynamic balance. J Orthop Sports Phys Ther 1998;27:356-60.

21. Shahbazi Moheb Seraj M, Sarrafzadeh J, Maroufi N, Ebrahimi Takamjani I, Ahmadi A, Negahban H. Comparison of postural balance between subgroups of nonspecific low-back pain patients Based on O'Sullivan classification system and normal subjects during lifting. Arch Bone Jt Surg 2019;7:52-60.

22. Foster NE, Hill JC, Hay EM. Subgrouping patients with low back pain in primary care: are we getting any better at it? Man Ther 2011;16:3-8.

23. Luomajoki H, Kool J, de Bruin ED, Airaksinen O. Reliability of movement control tests in the lumbar spine. BMC Musculoskelet Disord 2007;8:90.

24. O'Sullivan P. Diagnosis and classification of chronic low back pain disorders: maladaptive movement and motor control impairments as underlying mechanism. Man Ther 2005;10:242-55.

25. Dankaerts W, O'Sullivan P, Burnett A, Straker L, Davey P, Gupta R. Discriminating healthy controls and two clinical subgroups of nonspecific chronic low back pain patients using trunk muscle activation and lumbosacral kinematics of postures and movements: a statistical classification model. Spine (Phila Pa 1976) 2009;34:1610-8

26. O'Sullivan P. Classification of lumbopelvic pain disorders--why is it essential for management? Man Ther 2006;11:169-70.

27. Sheeran L, Sparkes V, Caterson B, Busse-Morris M, van Deursen R. Spinal position sense and trunk muscle activity during sitting and standing in nonspecific chronic low back pain: classification analysis. Spine (Phila Pa 1976) 2012;37:E486-95.

28. Hemming R, Sheeran L, van Deursen R, Sparkes V. Regional spinal kinematics during static postures and functional tasks in people with non-specific chronic low back pain. Int J Ther Rehabil 2015. doi: 10.12968/ijtr.2015.22.Sup8.S8b.

29. Hemming R, Sheeran L, van Deursen R, Sparkes V. Non-specific chronic low back pain: differences in spinal kinematics in subgroups during functional tasks. Eur Spine J 2018;27:163-70.

30. Dankaerts W, O'Sullivan PB, Straker LM, Burnett AF, Skouen JS. The inter-examiner reliability of a classification method for non-specific chronic low back pain patients with motor control impairment. Man Ther 2006;11:28-39.

31. Vibe Fersum K, O'Sullivan PB, Kvåle A, Skouen JS. Inter-examiner reliability of a classification system for patients with nonspecific low back pain. Man Ther 2009;14:555-61.

32. Onofrei RR, Amaricai E, Petroman R, Suciu O. Relative and ab- solute within-session reliability of the modified Star Excursion Balance Test in healthy elite athletes. PeerJ 2019;7:e6999.

33. Olmsted LC, Carcia CR, Hertel J, Shultz SJ. Efficacy of the Star Excursion Balance Tests in detecting reach deficits in subjects with chronic ankle instability. J Athl Train 2002;37:501-6.

34. Tsigkanos C, Gaskell L, Smirniotou A, Tsigkanos G. Static and dynamic balance deficiencies in chronic low back pain. J Back Musculoskelet Rehabil 2016;29:887-93.

35. Gribble PA, Hertel J, Plisky P. Using the Star Excursion Balance Test to assess dynamic postural-control deficits and outcomes in lower extremity injury: a literature and systematic review. J Athl Train 2012;47:339-57.

36. Appiah-Dwomoh EK, Müller S, Hadzic M, Mayer F. Star Excursion Balance Test in young athletes with back pain. Sports (Basel) 2016;4:E44.

37. Gribble PA, Hertel J. Considerations for normalizing measures of the Star Excursion Balance Test. Meas Phys Educ Exerc Sci 2003;7:89-100.

38. Carpes FP, Reinehr FB, Mota CB. Effects of a program for trunk strength and stability on pain, low back and pelvis kinematics, and body balance: a pilot study. J Bodyw Mov Ther 2008;12: 22-30.

39. Behennah J, Conway R, Fisher J, Osborne N, Steele J. The relationship between balance performance, lumbar extension strength, trunk extension endurance, and pain in participants with chronic low back pain, and those without. Clin Biomech (Bristol, Avon) 2018;53:22-30.

40. Mergner T, Schweigart G, Maurer C, Blümle A. Human postural responses to motion of real and virtual visual environments under different support base conditions. Exp Brain Res 2005;167: 535-56.

41. Bray H, Moseley GL. Disrupted working body schema of the trunk in people with back pain. Br J Sports Med 2011;45:168-73.

42. Waddell G, Newton M, Henderson I, Somerville D, Main CJ. A Fear-Avoidance Beliefs Questionnaire (FABQ) and the role of fear-avoidance beliefs in chronic low back pain and disability. Pain 1993;52:157-68.

43. Hooper TL, James CR, Brismée JM, Rogers TJ, Gilbert KK, Browne KL, et al. Dynamic balance as measured by the YBalance Test is reduced in individuals with low back pain: a cross-sectional comparative study. Phys Ther Sport 2016;22:2934.

44. Fullam K, Caulfield B, Coughlan GF, Delahunt E. Kinematic analysis of selected reach directions of the Star Excursion Balance Test compared with the Y-Balance Test. J Sport Rehabil 2014;23:27-35.

45. Hemmati L, Rojhani-Shirazi Z, Malek-Hoseini H, Mobaraki I. Evaluation of static and dynamic balance tests in single and dual task conditions in participants with nonspecific chronic low back pain. J Chiropr Med 2017;16:189-94.

46. Dankaerts W, O'Sullivan P, Burnett A, Straker L. Differences in sitting postures are associated with nonspecific chronic low back pain disorders when patients are subclassified. Spine (Phila $\mathrm{Pa}$ 1976) 2006;31:698-704.

47. Madigan ML, Davidson BS, Nussbaum MA. Postural sway and joint kinematics during quiet standing are affected by lumbar extensor fatigue. Hum Mov Sci 2006;25:788-99. 